\title{
BMJ Open Management of shoulder pain by UK general practitioners (GPs): a national survey
}

\author{
Majid Artus, ${ }^{1}$ Danielle A van der Windt, ${ }^{1}$ Ebenezer K Afolabi, ${ }^{1}$ \\ Rachelle Buchbinder, ${ }^{2,3}$ Linda S Chesterton, ${ }^{1}$ Alison Hall, ${ }^{1}$ Edward Roddy, ${ }^{1}$ \\ Nadine E Foster ${ }^{1}$
}

To cite: Artus M, van der Windt DA, Afolabi EK, et al. Management of shoulder pain by UK general practitioners (GPs): a national survey. BMJ Open 2017;7:e015711. doi:10.1136/ bmjopen-2016-015711

- Prepublication history for this paper is available online. To view these files please visit the journal online (http://dx.doi. org/10.1136/bmjopen-2016015711).

Received 29 December 2016 Revised 13 April 2017 Accepted 3 May 2017

\section{CrossMark}

${ }^{1}$ Arthritis Research UK Primary Care Centre, Research Institute for Primary Care \& Health Sciences, Keele University, Keele, Staffordshire, UK

${ }^{2}$ Monash Department of Clinical Epidemiology, Cabrini Institute, Melbourne, Victoria, Australia

${ }^{3}$ Department of Epidemiology and Preventive Medicine,

Monash University, Melbourne, Victoria, Australia

Correspondence to

Dr Majid Artus; m.artus@keele. ac.uk

\section{ABSTRACT}

Objectives Studies in Canada, the USA and Australia suggested low confidence among general practitioners (GPs) in diagnosing and managing shoulder pain, with frequent use of investigations. There are no comparable studies in the UK; our objective was to describe the diagnosis and management of shoulder pain by GPs in the UK.

Methods A national survey of a random sample of 5000 UK GPs collected data on shoulder pain diagnosis and management using two clinical vignettes that described primary care presentations with rotator cuff tendinopathy (RCT) and adhesive capsulitis (AdhC).

Results Seven hundred and fourteen (14.7\%) responses were received. $56 \%$ and $83 \%$ of GPs were confident in their diagnosis of RCT and AdhC, respectively, and a wide range of investigations and management options were reported. For the RCT presentation, plain radiographs of the shoulder were most common $(60 \%)$, followed by blood tests (42\%) and ultrasound scans (USS) (38\%). $19 \%$ of those who recommended a radiograph and $76 \%$ of those who recommended a USS did so 'to confirm the diagnosis'. For the AdhC presentation, the most common investigations were blood tests $(60 \%)$, plain shoulder radiographs (58\%) and USS (31\%). More than two-thirds of those recommending a USS did so 'to confirm the diagnosis'. The most commonly recommended treatment for both presentations was physiotherapy (RCT 77\%, AdhC $71 \%$ ) followed by non-steroidal anti-inflammatory drugs (RCT 58\%, AdhC 74\%). 17\% opted to refer the RCT to secondary care (most often musculoskeletal interface service), compared with $31 \%$ for the AdhC.

Conclusions This survey of GPs in the UK highlights reliance on radiographs and blood tests in the management of common shoulder pain presentations. GPs report referring more than 7 out of 10 patients with RCT and AdhC to physiotherapists. These findings need to be viewed in the context of low response to the survey and, therefore, potential non-response bias.

\section{BACKGROUND}

Shoulder pain is common with an annual population prevalence of up to $46.7 \%$ and lifetime prevalence of up to $66.7 \% .^{12}$ It is mainly managed in primary care where it is the third most common musculoskeletal (MSK) reason

\section{Strengths and limitations of this study}

This is the first study to attempt to describe the diagnosisand management of shoulder pain patients by GPs inthe UK.

- A combination of genral questions and vignette based questions were used.

- The low response means that caution needs to be exercised before the results can be generalised to all GPs in the UK.

to consult, with up to $3 \%$ of adults likely to consult with new shoulder pain annually. ${ }^{13-5}$ The clinical management in primary care is based on the clinical history and physical examination to establish the likely clinical diagnosis, differentiating between the most common clinical causes such as rotator cuff tendinopathy (RCT), adhesive capsulitis (AdhC)/frozen shoulder, glenohumeral osteoarthritis (OA) and acromioclavicular joint (ACJ) disorders, and exclude referred neck pain and serious pathology. Surveys among primary care practitioners in Canada, ${ }^{6}$ the $\mathrm{USA}^{7}$ and Australia ${ }^{8}$ have shown high reliance on investigations in the management of shoulder pain. General practitioners (GPs) and other primary healthcare clinicians such as physiotherapists, face uncertainties and challenges in the diagnosis of presentations such as shoulder pain, ${ }^{9}{ }^{10}$ which might explain the over-reliance on investigations. ${ }^{11}$

It is not clear to what extent the findings from the surveys in Canada, the USA and Australia translate to the UK and its primary healthcare system, where the predominant model is one where GPs act as gatekeepers to other healthcare services. GP access to some investigations such as magnetic resonance imaging (MRI), ultrasound scan (USS) and plain radiographs is increasingly restricted in some areas in the UK. There are no comparable studies that explore the management 
of shoulder pain in primary care in the UK. One study, conducted more than 10 years ago, used data from medical records, ${ }^{3}$ and therefore information on GPs' reasoning for investigations, treatment and referral decisions was not available. It is likely that such estimates are an underestimation of care, compared with other methods such as survey questionnaires. ${ }^{12}$ Exploring management decisions made by GPs for shoulder pain in the UK is important to provide empirical evidence about the gap between practice and best evidence and to guide future research in this area. The primary aim of this study was therefore to describe the diagnoses and management of common shoulder pain presentations by GPs in the UK.

Postal questionnaires are the most commonly used distribution method to reach target populations in survey studies. Response among clinicians including GPs, however, is often low and rarely exceeds $30 \%$ in the UK. ${ }^{13}$ A number of advantages have been suggested for the use of online data distribution and collection methods, including lower cost, convenience and timesaving for both participants and researchers, and ease with which complete and correct data can be collected. ${ }^{15}$ However, response to online methods among GPs is variable, ranging from $1 \%{ }^{16}$ to $40 \%,{ }^{17}$ and currently it is not clear whether GPs in the UK respond better to postal or online surveys. Therefore, a secondary aim of this study was to compare the response from GPs to postal and online (email) survey distribution methods.

\section{METHODS}

A cross-sectional descriptive survey that includes general questions (on the diagnosis and management of shoulder pain) and specific questions related to clinical vignettes describing the two most common shoulder pain presentations in primary care was conducted between April and July 2015. The first vignette described a presentation typical in general practice of RCT, while the second vignette described early AdhC (see Box 1). The vignettes were adapted from previous surveys ${ }^{6-8}$ to facilitate comparison. Only two vignettes were chosen to minimise the burden on GPs and to try to optimise response.

The questionnaire was informally piloted among a small group of GPs in our research institute to assess clarity, acceptability, clinical relevance, representation of real patients and time required for completion, which was estimated to be around 5-10 mins. The questionnaire included three main sections:

A. Participants' characteristics: gender, duration of clinical experience as a GP whether a GP with special interest (GPwSI) in MSK medicine (GPs who have obtained a formal diploma in MSK medicine and who practice in a specialised MSK services), postgraduate training on shoulder pain or MSK pain that included shoulder pain (eg, workshops, online training modules, postgraduate degrees in MSK medicine and so on), size of practice (number of GPs in the practice), location of practice (inner city (urban), rural and semirural) and type of employment (GP principal, salaried GP, locum GP or other). In the UK, a GP principal is a vocationally trained GP who has full General Medical Council registration and is contracted by a local health authority or health board to provide general medical services to patients without supervision. ${ }^{18}$ A group of GP principals in a practice form a GP partnership, which is the historic model of general practice in the UK in which the partner undertakes all aspects of the practice including clinical and business aspects. In recent years, other models of working include salaried GPs who are contracted by the practice to undertake agreed aspects of clinical work.

B. Vignette-based questions: for each of the clinical vignettes:

1. Diagnosis: GPs were asked to select what they thought the clinical diagnosis was from a list of options: acute rotator cuff tear, glenohumeral OA, ACJ disorders, RCT, referred neck pain, AdhC and a free-text option. For each diagnosis selected they were asked to rate their confidence in the diagnosis on a 7-point Likert-type scale ('definitely yes', 'most likely', 'likely', 'not sure', 'unlikely', 'most unlikely' and 'definitely not').

2. Investigations: GPs were asked to state whether they would request investigations for each patient. If yes, they were asked to select from a list of options including blood tests, USS of the shoulder, plain radiograph of the shoulder, MRI of the shoulder, MRI of the cervical spine and a free-text option. They were also asked to select a reason for any investigation they selected, choosing from the following options: 'to confirm the diagnosis', 'to exclude other diagnoses', 'to guide treatment options', 'to decide on a specialist referral' and a free-text option. They were free to select any combination of investigations but restricted to one reason only for each investigation.

3. Treatment: GPs were asked to indicate whether they would recommend treatment. If yes, they were asked to select from a list of options: analgesic medications (including paracetamol, non-steroidal anti-inflammatory drugs (NSAIDs) and so on), corticosteroid shoulder injection (USS guided or not) or physiotherapy and a free-text option. They were free to select any combination of options.

4. Referral to a specialist: GPs were asked to indicate whether they would refer the patient for a specialist opinion. If yes, they were asked to select from a list of referral destination options: MSK service clinic or equivalent, rheumatology or orthopaedics, and a free-text option. They were free to select any combination of options. 
C. General questions: these questions were unrelated to any specific clinical vignette and aimed to assess GP decision making regarding requesting ultrasound and MRI scans and treatment decisions. GPs were asked to 'give an estimate of the percentage of patients presenting with shoulder pain for whom (they) offer any of the following management options, at any time during their episode of shoulder pain': investigations (shoulder USS, MRI), prescribing analgesic medications, recommending corticosteroid shoulder injection, referral to physiotherapy and referral to a specialist service. Responders were free to enter any percentage figure for each option, from $0 \%$ to $100 \%$.

\section{Survey distribution methods}

A random sample of 5000 UK GPs was selected from Binley's database ${ }^{19}$ which has been identified as the most comprehensive source of contact details and basic demographic information for UK GPs. A postal questionnaire was sent to a randomly selected 2500 GPs and an email containing a web link to an online questionnaire sent to the remaining 2500. Equal distribution from the four UK countries (England, Scotland, Wales and Northern Ireland) was ensured across the two samples. For the postal survey, each GP was sent a study pack that included a letter introducing the study and an invitation to participate, the study questionnaire and a return stamped addressed envelope. After 2 weeks, non-responders were sent a postcard reminder and after a further 2 weeks they were sent a second reminder with a full study pack. For the online method, GPs were sent an email using a similar letter introducing the study and inviting them to participate through a link to an online questionnaire using SurveyMonkey. ${ }^{20}$ After 2 weeks, non-responders were sent a reminder email and after a further 2 weeks they were sent a further and final email with the link to the online questionnaire. The online questionnaire was designed in a way that included mandatory fields for all relevant areas and did not allow erroneous data entry (eg, numbers where text is required), comments or free text other than in the specified fields. These design arrangements were not possible in the postal questionnaire.

\section{Sample size}

With the total number of UK GPs is estimated to be over $46000,{ }^{21}$ to have a $5 \%$ margin of sampling error for the variable of selecting the correct diagnosis for each patient described in the clinical vignettes and 99\% confidence level, 660 completed questionnaires were required. Based on responses obtained in previous similar surveys, an overall response of $14 \%$ was anticipated, $20 \%$ for the postal survey ${ }^{81314}$ and $4 \%$ for the online survey. ${ }^{1617} \mathrm{~A}$ total of 5000 questionnaires were required to be sent.

\section{Analysis}

Descriptive analyses were used to summarise the confidence in making the diagnoses, investigations selected and the reasons for selecting them, and treatment options and referrals to specialist care. To summarise the confidence in the diagnoses, responses to the seven-item

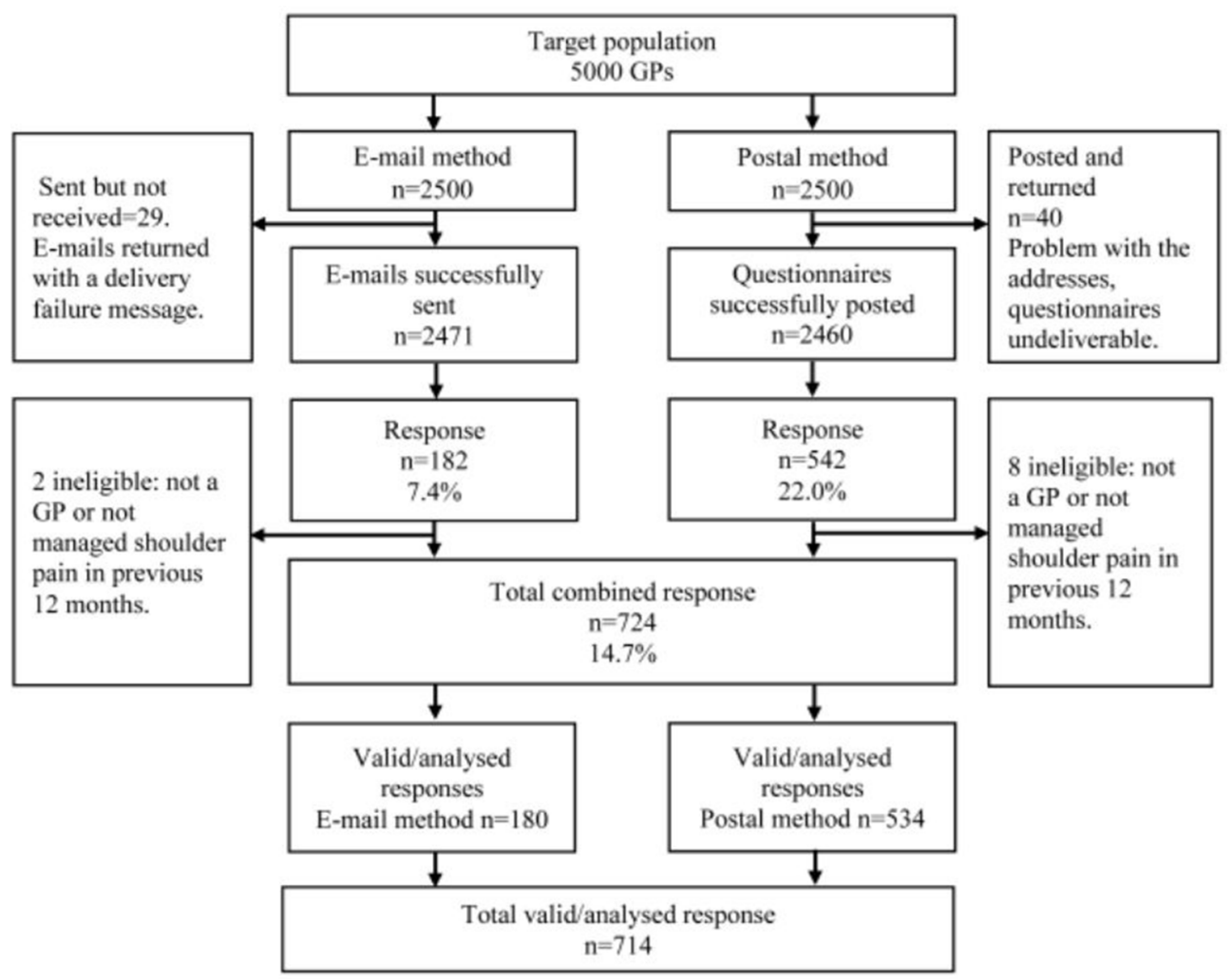

Figure 1 Survey flowchart. 
Likert scale were converted into five items, by combing the responses 'definitely yes' and 'most likely' into one group ('confident yes'), and 'definitely not' and 'most unlikely' into another group ('confident no'). The remaining responses of 'likely', 'not sure' and 'unlikely' were left ungrouped. Percentage responses to the general questions were categorised into five groups: $0 \%-10 \%$ : 'rare use'; $11 \%-25 \%$ : 'low use'; 26\%-50\%: 'moderate use'; $51 \%-75 \%$ : 'regular use' and 67\%-100\%: 'routine use'.

Subgroup analyses were undertaken to assess whether selecting investigations was associated with confidence in the diagnosis. Responses to investigations, treatments, referrals and diagnoses were also analysed separately for GPwSI in MSK medicine or GPs who had postgraduate MSK training compared with the rest of the participants to assess the impact of knowledge and experience in MSK medicine on management decisions.

\section{RESULTS}

Of 2500 postal questionnaires mailed, 2460 were successfully delivered and 542 returned completed (adjusted response $22.0 \%$ ). In the online survey, emails to 29 GPs were returned due to incorrect addresses and of the remaining 2471 GPs, 182 online questionnaires were completed (adjusted response 7.4\%). The combined overall adjusted response was $14.7 \%$. Of these 724 responses, 10 were deemed ineligible (not GPs or GPs who had not managed a patient with shoulder pain in previous 12 months) and 714 were used in the analysis (figure 1).

\section{Participants' characteristics}

Responders versus non-responders

There were slightly more male responders $(410 / 714$ $(57 \%))$ than non-responders $(2159 / 4286(50 \%))$. A smaller proportion of responders than non-responders were from England (507 (71\%) vs $3679(86 \%)$ ) and a larger proportion from Scotland (106 (15\%) vs 235 $(6 \%))$, and proportions of responders and non-responders were similar in Wales $(57(8 \%)$ vs $267(6 \%))$ and Northern Ireland (45 (6\%) vs $105(3 \%))$. No data on other characteristics were available for further comparison between responders and non-responders.

\section{Responders' characteristics}

The majority of responders were GP principals $(531,74 \%)$ with a mean duration of clinical practice of 17.5 years (SD 12.1). Most worked in practices of 5-10 GPs $(466,66 \%)$ and were based in semirural (47\%) or inner city (urban) $(42 \%)$ rather than rural locations $(10 \%)$. Forty-seven responders (6\%) were GPwSIs in MSK medicine, and 265 $(37 \%)$ had previous postgraduate training on shoulder pain or MSK pain that included shoulder pain. Of the 227 participants who provided information on the type of MSK training, the majority $(147,65 \%)$ received workshop-based training, followed by online training $(22,3 \%)$ and postgraduate degree $(18,3 \%)$.
Table 1 Participants' characteristics by type of survey, number (\%) provided unless stated otherwise

\begin{tabular}{|c|c|c|c|}
\hline & \multirow[b]{2}{*}{$\begin{array}{l}\text { Total } \\
\mathrm{n}=714\end{array}$} & \multicolumn{2}{|c|}{ Survey type } \\
\hline & & $\begin{array}{l}\text { Online } \\
\mathrm{n}=180\end{array}$ & $\begin{array}{l}\text { Postal } \\
n=534\end{array}$ \\
\hline Gender: male & $410(57)$ & $131(73)$ & $279(53)$ \\
\hline \multicolumn{4}{|l|}{ GP type } \\
\hline GP principal & $531(74)$ & $171(95)$ & $360(68)$ \\
\hline Salaried GP & $151(21)$ & $6(3)$ & $145(27)$ \\
\hline Locum GP & $21(3)$ & $2(1)$ & $19(4)$ \\
\hline Other & $9(1)$ & $1(0.6)$ & $8(2)$ \\
\hline \multicolumn{4}{|c|}{ Duration since qualification as a GP, years } \\
\hline Mean (SD) & $18(12)$ & $30(5)$ & $13(11)$ \\
\hline Median & 12 & 29.5 & 8 \\
\hline Range & $1-55$ & $21-47$ & $1-55$ \\
\hline \multicolumn{4}{|l|}{ Practice location/type } \\
\hline Inner city (urban) & $301(42)$ & $83(46)$ & $218(41)$ \\
\hline Semirural & $336(47)$ & $84(47)$ & $252(48)$ \\
\hline Rural & $73(10)$ & $13(7)$ & $60(11)$ \\
\hline \multicolumn{4}{|l|}{ Number of GPs in practice } \\
\hline Single-handed & $5(1)$ & $2(1)$ & $3(1)$ \\
\hline 2-4 GPs & $169(24)$ & $20(11)$ & $149(28)$ \\
\hline 5-10 GPs & $466(66)$ & $137(76)$ & $329(62)$ \\
\hline$>10 \mathrm{GPs}$ & $68(10)$ & $21(12)$ & $47(10)$ \\
\hline $\begin{array}{l}\text { GPwSI MSK medicine } \mathrm{n} \\
\text { (\%) }\end{array}$ & $47(6)$ & $16(9)$ & $31(6)$ \\
\hline \multicolumn{4}{|l|}{ MSK training } \\
\hline Received training & $265(37)$ & $72(40)$ & $193(36)$ \\
\hline $\begin{array}{l}\text { Responses to training } \\
\text { type* }\end{array}$ & $227(86)$ & & \\
\hline Online only & $22(3)$ & & \\
\hline Workshop only & $147(21)$ & & \\
\hline Postgraduate only & $18(3)$ & & \\
\hline Online and workshops & $36(5)$ & & \\
\hline $\begin{array}{l}\text { Online and } \\
\text { postgraduate }\end{array}$ & $1(0.1)$ & & \\
\hline $\begin{array}{l}\text { Workshop and } \\
\text { postgraduate }\end{array}$ & $1(0.1)$ & & \\
\hline $\begin{array}{l}\text { Online and workshop } \\
\text { and postgraduate }\end{array}$ & $2(0.3)$ & & \\
\hline
\end{tabular}

*Type of MSK training not described separately for the two subgroups because of low numbers.

GP, general practitioner; GPwSI, GP with special interest; MSK, musculoskeletal.

Compared with the postal method, responders to the online survey were more likely to be male GP principals with a longer duration of clinical practice (table 1). Due to the design features, the online responses were complete and did not include any erroneous or missing data. This contrasts with the postal responses that included some 
Table 2 GPs confidence in diagnosis for the RCT clinical vignette

\begin{tabular}{lllllll}
\hline & RCT (n\%) & $\begin{array}{l}\text { Glenohumeral } \\
\text { OA (n\%) }\end{array}$ & $\begin{array}{l}\text { ACJ } \\
\text { disorders (n\%) }\end{array}$ & AdhC (n\%) & $\begin{array}{l}\text { Acute RC } \\
\text { tear (n\%) }\end{array}$ & $\begin{array}{l}\text { Referred neck } \\
\text { pain (n\%) }\end{array}$ \\
\hline Confident yes & $379(56)$ & $80(13)$ & $55(9)$ & $60(10)$ & $24(4)$ & $2(0.3)$ \\
Likely & $173(26)$ & $227(35)$ & $127(20)$ & $88(14)$ & $56(9)$ & $44(7)$ \\
Uncertain & $64(10)$ & $99(15)$ & $111(18)$ & $72(12)$ & $73(12)$ & $64(11)$ \\
Unlikely & $48(7)$ & $174(27)$ & $240(39)$ & $224(36)$ & $277(45)$ & $336(55)$ \\
Confident no & $8(1)$ & $62(10)$ & $89(14)$ & $183(29)$ & $185(0)$ & $165(27)$ \\
Total & 672 & 642 & 622 & 627 & 615 & 611 \\
\hline
\end{tabular}

ACJ, acromioclavicular joint; AdhC, adhesive capsulitis; GPs, general practitioners; OA, osteoarthritis; $R C$, rotator cuff; RCT, rotator cuff tendinopathy.

missing data across all questions. The proportion of missing data ranged from $1 \%$ to $29 \%$. The questions with the largest proportion of missing data included the destination of referrals to specialist care (29\% for the RCT, $13 \%$ for the AdhC vignettes) and the specific type of postgraduate training in MSK medicine (14\%).

\section{Clinical scenario one: RCT}

Diagnosis

Just over half of the responders who selected the correct diagnosis of RCT indicated they were confident of this diagnosis $(379,56 \%) ; 64(10 \%)$ stated they were not sure, and $173(26 \%)$ stated the diagnosis was likely (table 2). Between 64 and $99(61 \%-77 \%)$ were uncertain of the likelihood of any other diagnosis.

Investigations

Of the 702 GPs who provided responses, 296 (42\%) did not select any investigation. Of the remainder, the most common investigation selected was a plain radiograph of the shoulder, selected by $242(60 \%)$ (table 3$)$. Blood tests were the second most frequently selected, by 172 GPs (42\%), followed by USS of the shoulder, selected by 154 GPs $(38 \%)$. The majority selected these investigations in combination with others, while only $40 \%, 23 \%$ and $48 \%$, respectively, selected them on their own. The most common reason for selecting USS and MRI of the shoulder for this vignette was to confirm the diagnosis; for plain radiograph of the shoulder and blood tests was to exclude other diagnoses.
Linking confidence in diagnosis with investigations, figure 2 shows that regardless of the diagnosis and the confidence in selecting the diagnosis, the majority of responders requested investigations. There was no pattern or association with how confident or not the responders were in making any diagnosis. Four of the 27 GPs $(15 \%)$ who were confident that the correct diagnosis was RCT and selected plain radiograph of the shoulder gave the reason as 'to confirm the diagnosis'. The same reason was given by 16 of the 93 GPs (17\%) who said RCT was a 'likely' diagnosis.

\section{Treatment and referral to specialist care}

Physiotherapy was the most commonly selected treatment $(526 / 682,77 \%)$, followed by NSAIDs $(396,58 \%$ ) and corticosteroid shoulder injection (330, 48\%). The majority of GPs selected more than one treatment option. The most common combination was NSAIDs and physiotherapy $(306,45 \%)$ followed by corticosteroid shoulder injection and physiotherapy $(245,36 \%)$.

One hundred and twenty-three out of 705 GPs (18\%) said they would refer the patient described in this vignette to a specialist in secondary care. Specific destinations were provided by 87 respondents; the most common was an MSK interface clinic or equivalent $(79,91 \%)$ followed by orthopaedics $(5,6 \%)$ and rheumatology $(3,3 \%)$.

GPwSI in MSK medicine and GPs who reported MSK/ shoulder postgraduate training were similar in that both

Table 3 Selected investigations for the RCT clinical vignette

\begin{tabular}{|c|c|c|c|c|c|c|}
\hline $\begin{array}{l}\text { Reason for requesting } \\
\text { investigation }\end{array}$ & $\begin{array}{l}\text { Plain radiograph } \\
\text { shoulder }(\mathbf{n} \%)\end{array}$ & $\begin{array}{l}\text { Blood } \\
\text { tests }(n \%)\end{array}$ & $\begin{array}{l}\text { USS } \\
\text { shoulder (n\%) }\end{array}$ & $\begin{array}{l}\text { Plain } \\
\text { radiograph } \\
\text { neck }(\mathrm{n} \%)\end{array}$ & $\begin{array}{l}\text { MRI } \\
\text { shoulder (n\%) }\end{array}$ & $\begin{array}{l}\text { MRI } \\
\text { neck (n\%) }\end{array}$ \\
\hline To confirm the diagnosis & $59(24)$ & $8(5)$ & $83(54)$ & $5(24)$ & $11(55)$ & 0 \\
\hline To exclude other diagnoses & $125(52)$ & $154(90)$ & $5(3)$ & $9(43)$ & $2(10)$ & $2(50)$ \\
\hline To guide treatment option & $32(13)$ & $3(2)$ & $32(21)$ & $2(10)$ & 0 & 0 \\
\hline To decide whether to refer on & $4(2)$ & 0 & $7(5)$ & $2(10)$ & $5(25)$ & $1(25)$ \\
\hline Total & 242 & 172 & 154 & 21 & 20 & 4 \\
\hline
\end{tabular}

$R C T$, rotator cuff tendinopathy; USS, ultrasound scan. 


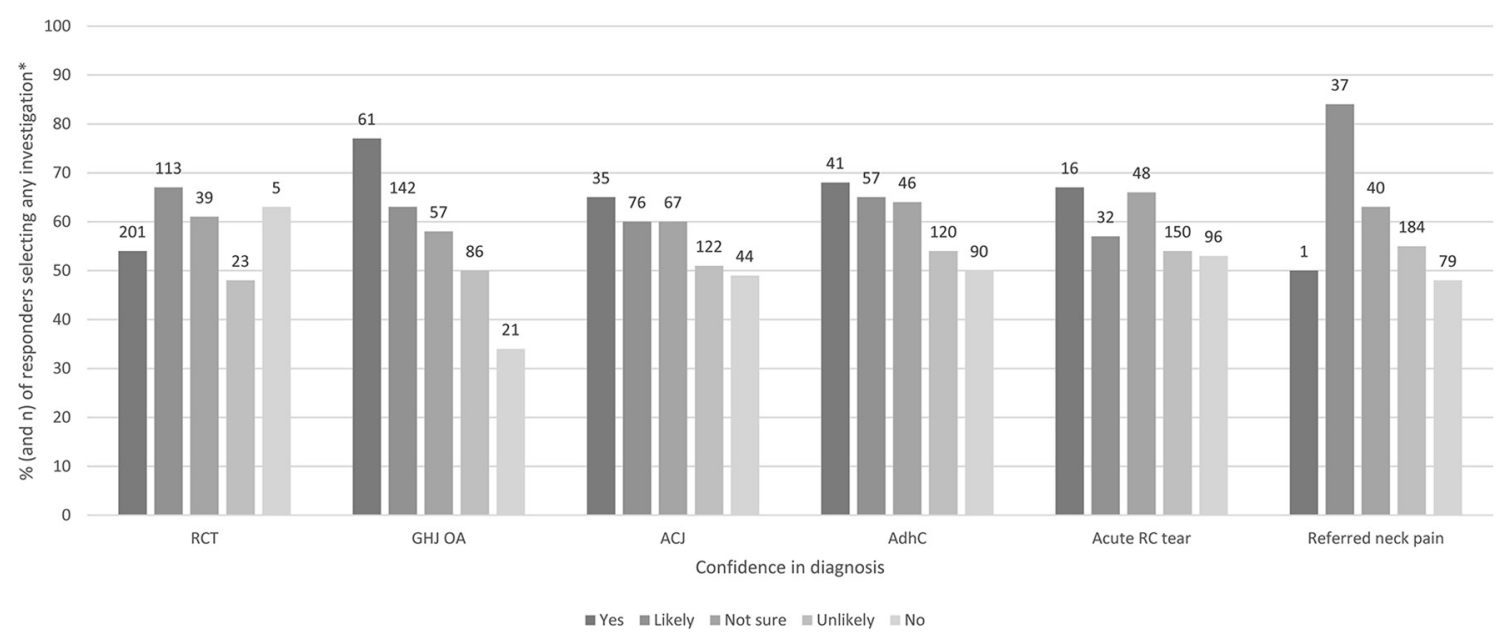

Figure 2 Confidence in diagnoses for clinical vignette one (rotator cuff tendinopathy) and percentage and number of responders selecting any investigation for each diagnosis. ${ }^{*} \%$ percentage of responders who selected investigation for each confidence category for each diagnosis. ACJ acromioclavicular joint, AdhC adhesive capsulities, GHJ OA gleno-humeral joint osteoarthritis, RC rotator cuff, RCT rotator cuff tendinopathy.

groups were more likely to select corticosteroid shoulder injection $(32,68 \%$ vs $298,47 \%)$ and less likely to select NSAID medication (19, $40 \%$ vs $375,59 \%)$, respectively, and the differences were statistically significant ( $\mathrm{p}=0.005$ and $\mathrm{p}=0.012$, respectively). There were no other differences between these groups.

\section{Clinical scenario two:early AdhC Diagnosis}

The majority of GPs indicated that they were confident of the correct diagnosis of the patient described in this vignette $(565 / 683,83 \%)$ (table 4 ) with $25(4 \%)$ stating they were not sure and $63(9 \%)$ stating that the diagnosis was likely. Similar to the previous vignette, between 58 and $105(10-17 \%) \%)$ were 'not sure' about any of the other diagnoses.

\section{Investigations}

Of the 694 GPs who provided responses, 297 (43\%) did not select any investigation. Of the remainder, $238(60 \%)$ selected blood tests (table 5), followed by a plain radiograph of the shoulder $(232,58 \%)$ and USS of the shoulder $(122,31 \%)$. The majority selected these in combination with other investigations, and only $26 \%, 27 \%$ and $31 \%$, respectively, selected them on their own. The only other investigation suggested was plain chest radiograph, by three responders. The most common reasons for selecting investigations are similar to those related to the first vignette: for USS and MRI of the shoulder to confirm the diagnosis, and for blood tests and plain radiographs of the shoulder to exclude other diagnoses.

Figure 3 shows the link between confidence in diagnosis and investigations and, similar to vignette one, it shows that regardless of the diagnosis and the confidence in selecting the diagnosis, the majority of responders requested investigations. There was no pattern or association with how confident or not the responders were in making any diagnosis. Proportions of responders requesting investigations of the GPs who were confident in the diagnosis of AdhC, the following gave the reason as to 'confirm the diagnosis': 25 of 177 (14\%) who selected blood tests, 38 of $168(23 \%)$ who selected a plain radiograph of the shoulder and 59 of the $74(80 \%)$ who selected USS of the shoulder.

\section{Treatment and referral to specialist care}

NSAIDs were the most commonly selected treatment $(504 / 680,74 \%)$, followed by physiotherapy $(482,71 \%)$ then corticosteroid shoulder injection $(330,49 \%)$. The

Table 4 GPs confidence in diagnosis for the AdhC clinical vignette

\begin{tabular}{lllllll} 
& AdhC (n\%) & RCT (n\%) & Acute RC tear $(\mathbf{n} \%)$ & $\begin{array}{l}\text { Glenohumeral } \\
\text { OA (n\%) }\end{array}$ & $\begin{array}{l}\text { ACJ } \\
\text { disorders (n\%) }\end{array}$ & $\begin{array}{l}\text { Referred neck } \\
\text { pain (n\%) }\end{array}$ \\
\hline Definitely yes & $565(83)$ & $46(8)$ & $45(7)$ & $13(2)$ & $3(1)$ & $6(1)$ \\
Likely & $63(9)$ & $162(26)$ & $70(12)$ & $76(12)$ & $36(6)$ & $26(4)$ \\
Uncertain & $25(4)$ & $105(17)$ & $74(12)$ & $83(14)$ & $61(10)$ & $58(10)$ \\
Unlikely & $28(4)$ & $213(35)$ & $265(43)$ & $307(51)$ & $330(55)$ & $313(53)$ \\
Definitely not & $2(0.02)$ & $87(14)$ & $155(25)$ & $125(21)$ & $168(28)$ & $189(32)$ \\
Total & 683 & 613 & 610 & 604 & 598 & 592 \\
\hline
\end{tabular}

$A d h C$, adhesive capsulitis; GPs, general practitioners; OA, osteoarthritis; $R C$, rotator cuff; $R C T$, rotator cuff tendinopathy. 
Table 5 Selected investigations for the early AdhC clinical vignette

\begin{tabular}{|c|c|c|c|c|c|c|}
\hline $\begin{array}{l}\text { Reason for requesting } \\
\text { investigation }\end{array}$ & $\begin{array}{l}\text { Blood } \\
\text { tests }(n \%)\end{array}$ & $\begin{array}{l}\text { Plain } \\
\text { radiograph } \\
\text { shoulder (n\%) }\end{array}$ & $\begin{array}{l}\text { USS } \\
\text { shoulder (n\%) }\end{array}$ & $\begin{array}{l}\text { MRI } \\
\text { shoulder (n\%) }\end{array}$ & $\begin{array}{l}\text { Plain } \\
\text { radiograph } \\
\text { neck }(\mathbf{n} \%)\end{array}$ & $\begin{array}{l}\text { MRI } \\
\text { neck (n\%) }\end{array}$ \\
\hline To confirm the diagnosis & $36(15)$ & $54(23)$ & $83(68)$ & $26(72)$ & 9 (39) & $9(60)$ \\
\hline To exclude other diagnoses & $177(74)$ & $151(65)$ & $12(10)$ & $6(17)$ & $11(48)$ & $4(27)$ \\
\hline To guide treatment option & $14(6)$ & $8(4)$ & $9(7)$ & $2(6)$ & $2(87)$ & $1(7)$ \\
\hline Total number & 238 & 232 & 122 & 36 & 23 & 15 \\
\hline
\end{tabular}

AdhC, adhesive capsulitis; USS, ultrasound scans, MRI, magetic resonance imaging.

majority of responders selected more than one treatment. The most common combination was NSAIDs and physiotherapy $(355,52 \%)$ followed by corticosteroid shoulder injection and physiotherapy $(253,37 \%)$.

Two hundred and eighteen out of 698 GPs (31\%) stated that they would refer the patient to a secondary care specialist. Of 189 who specified a referral destination, the most common was an MSK interface clinic or equivalent $(141,75 \%)$, orthopaedics $(40,21 \%)$ and rheumatology $(8,4 \%)$.

GPwSIs in MSK medicine were more likely to select plain radiographs of the shoulder $(24,77 \%$ vs 206 , $57 \%, \mathrm{p}=0.024$ (only 230 GPs provided such information) ) and corticosteroid shoulder injection (30, 64\% vs $299,47 \%, \mathrm{p}=0.03$ (only 329 GPs provided such information) ) and less likely to select NSAIDs $(26,55 \%$ vs $477,76 \%, \mathrm{p}=0.002$ (only 503 GPs provided such information)) than non-GPwSIs. GPs who had postgraduate MSK/shoulder training were less likely to request USS $(32,23 \%$ vs $90,35 \%, \mathrm{p}=0.015)$ but more likely to select corticosteroid shoulder injection $(145,57 \%)$ vs 184 ,
$43 \%, \mathrm{p}=0.00)$. There were no other differences between the groups.

\section{General questions on common management decisions}

Table 6 shows that in contrast to responses to the vignettebased questions, the majority of responders to the general questions $(394 / 687,57 \%)$ reported 'rarely' using USS for shoulder pain. Pain medications were prescribed routinely by the majority of GPs for shoulder pain (604, $87 \%$ ). Corticosteroid shoulder injections and referral for physiotherapy were reported relatively frequently (by $264(38 \%)$ and $281(40 \%)$ of responders). Referrals to specialist care were reported as rare.

GPwSIs in MSK medicine and GPs with postgraduate MSK/shoulder training were more likely to provide corticosteroid shoulder injections, with statistically significant differences $(32 / 47,68 \%$ vs $298 / 653,47 \%, \mathrm{p}=0.03$ and $154 / 265,60 \%$ vs $176 / 447,41 \% \mathrm{p}=0.000$, respectively). Both GPwSIs in MSK medicine and GPs with postgraduate MSK/shoulder training were less likely to refer patients with shoulder pain for specialist care.

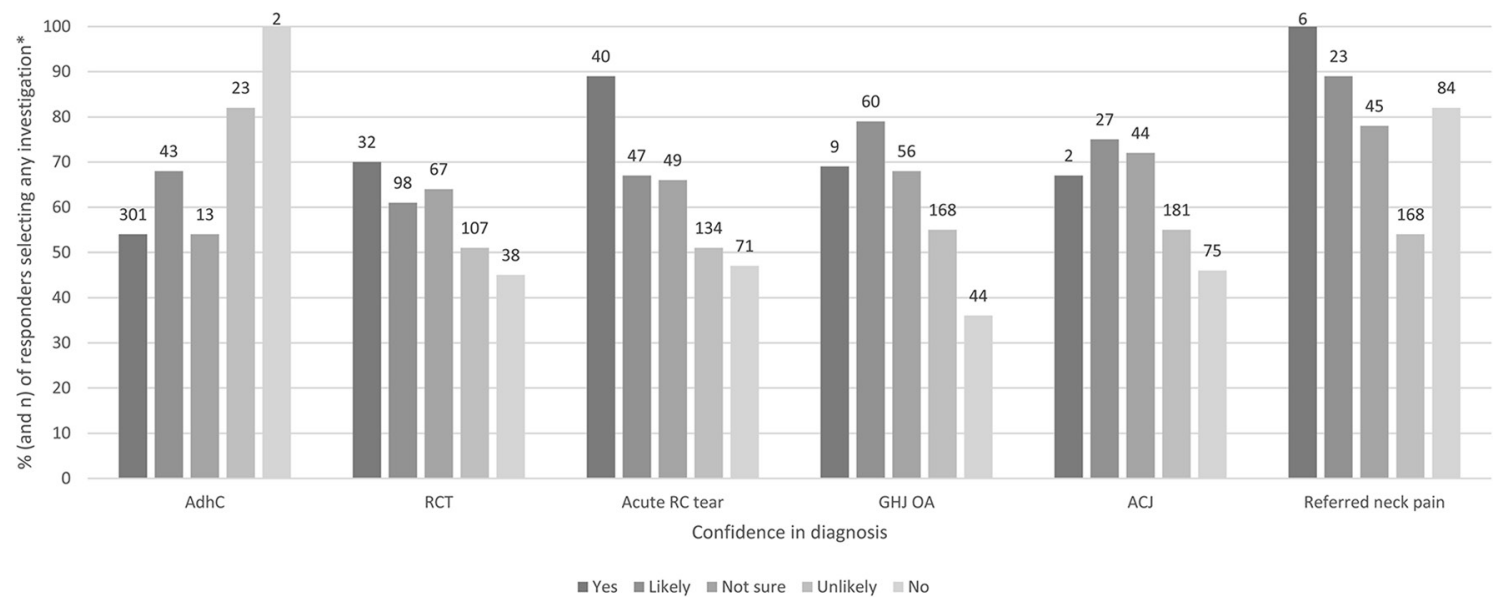

Figure 3 Confidence in diagnoses for clinical vignette two (early adhesive capsulitis) and percentage and number of responders selecting any investigation for each diagnosis. ${ }^{*} \%$ percentage of responders who selected investigation for each confidence category for each diagnosis. ACJ acromioclavicular joint, AdhC adhesive capsulities, GHJ OA gleno-humeral joint osteoarthritis, RC rotator cuff, RCT rotator cuff tendinopathy. 
Table 6 Frequency of common management options including imaging investigations and referrals

\begin{tabular}{|c|c|c|c|c|c|c|c|}
\hline & & USS (n\%) & MRI (n\%) & Pain medications ( $\mathrm{n} \%)$ & $\begin{array}{l}\text { Corticosteroid } \\
\text { shoulder } \\
\text { injection (n\%) }\end{array}$ & $\begin{array}{l}\text { Physiotherapy } \\
\text { (n\%) }\end{array}$ & $\begin{array}{l}\text { MSK and } \\
\text { specialist } \\
\text { referral }(\mathrm{n} \%)\end{array}$ \\
\hline Rare & $(0 \%-10 \%)$ & $394(57)$ & $638(94)$ & $5(0.7)$ & $179(26)$ & $34(5)$ & $310(45)$ \\
\hline Low & $(11 \%-25 \%)$ & $120(18)$ & $29(4)$ & $4(0.6)$ & $175(25)$ & 67 (10) & $180(26)$ \\
\hline Moderate & $(26 \%-50 \%)$ & $131(19)$ & $14(2)$ & $42(6)$ & $264(38)$ & $281(40)$ & $172(25)$ \\
\hline Regular & $(51 \%-75 \%)$ & $31(5)$ & 0 & $43(6)$ & $49(7)$ & $166(24)$ & $21(4)$ \\
\hline Routine & $(76 \%-100 \%)$ & $11(1)$ & $1(0.1)$ & $604(87)$ & $27(4)$ & $148(21)$ & $11(2)$ \\
\hline Total & & 687 & 682 & 698 & 694 & 696 & 694 \\
\hline
\end{tabular}

MSK, musculoskeletal; USS, ultrasound scan, MRI magnetic resonance imaging

\section{DISCUSSION}

This is the first national study to report on management of patients with shoulder pain by GPs in the UK, using clinical vignettes describing the most common shoulder pain presentations. Responses showed a wide variability in the investigation and treatment options selected. When responding to questions related to the vignettes, GPs appeared to rely heavily on investigations in the expectation that these would confirm the diagnosis or exclude other diagnoses.

The relatively modest proportions of respondents making the correct diagnosis for the clinical vignettes might represent low confidence in making a specific diagnosis, similar to findings from a previous study conducted in the Netherlands. ${ }^{11}$ Alternatively, it might be argued that this reflects natural reluctance to commit to a specific diagnosis based on limited (clinical) information provided in the clinical vignettes rather than genuine low confidence in making the diagnosis. However, this reluctance to commit strongly to the correct diagnosis was also associated with high investigation rate and variability of management decisions, which might mean that genuine inability to make a diagnosis cannot be completely ruled out as the reason. Even when GPs indicated they were confident in the diagnosis, they requested investigations to confirm the diagnosis, which seems to strengthen a theme of apparent reluctance of GPs to rely solely on clinical assessment to make a diagnosis.

The rationale for some choices of investigations is not clear. For the RCT vignette for instance, one in five of the GPs who were confident of the correct diagnosis selected a plain radiograph of the shoulder to confirm the diagnosis, a rationale that is not supported by published evidence. ${ }^{22-26}$ In the case of the early AdhC vignette, blood tests and radiography are also not usually indicated as part of a primary care assessment in the absence of red flags or indications for malignancy, polymyalgia rheumatic or diabetes, although it might be reasonable to request a blood test to identify people with undiagnosed diabetes because of its known strong association with AdhC. ${ }^{27}$ Unilateral shoulder pain without other symptoms makes polymyalgia rheumatica and rheumatoid arthritis unlikely, while the middle-aged woman in the vignette also suggests that glenohumeral joint OA is also unlikely as this usually affects older people. The frequency with which investigations were selected by GPs in response to the two vignettes contrasts with the much lower rate when the question about investigations was asked more generally. The reasons for this disparity are not clear, notwithstanding the possible role of the limited information provided in the vignettes, which reflects common shoulder pain presentation in general practice. Perhaps recall bias explains the lower rates reported in the general questioning, while responses to the vignettes may have been influenced by GPs intending to appear thorough in their approach to making a diagnosis. Some investigations, such as MRI scans, were rarely requested, irrespective of how the question was asked.

Although the clinical diagnosis for shoulder pain can be made based on clinical history and examination, special clinical diagnostic tests have low diagnostic utility. ${ }^{28}$ The role and utility of imaging, such as plain radiography, USS and MRI in primary care, is also considered to be limited. ${ }^{22-26}$ The link between clinical presentation and findings on imaging investigations and the relevance of some of those findings are not clear. Abnormalities identified on USS of the shoulder such as tendinopathy or partial tear may not be clinically relevant or associated with presenting symptoms, ${ }^{29}$ and identifying them as the target for treatment may lead to unnecessary anxiety for patients and potentially unnecessary intervention(s). There has been a steep rise in the number of referrals for surgical interventions for RCT despite a lack of evidence for significant benefit compared with conservative treatment options such as exercise. ${ }^{30}$ It is unclear whether this steep rise in surgical interventions is a consequence of increasing requests for imaging. The potential consequences of unnecessary investigations and interventions for patients and the healthcare systems are important and merit further investigation.

Although decisions regarding treatment, including referrals to secondary care, varied among GPs, NSAIDs and referral for physiotherapy were consistently and commonly recommended for both vignettes and irrespective of how the questions were asked (it is worth noting that the RCT vignette stated that the patient was already 
using paracetamol). GPs in the UK do not appear to use other treatments such as oral steroids and hydrodistension for which evidence of effectiveness for early AdhC exists. ${ }^{31}{ }^{32}$ GPs' treatment decisions were broadly in line with best available evidence for the management of these two clinical conditions and shoulder pain in general ${ }^{2633} 34$ taking into account benefit and safety of the treatment in general practice. Although we did not ask specifically about combination of treatments, GPs commonly reported offering combined treatments. Such treatment combinations need to be more carefully tested to see whether they provide greater benefit for patients.

A minority of GPs considered a referral to a specialist in secondary care, in particular for the early AdhC patient, while referrals to MSK interface clinics were more frequently proposed. In the UK, the provision of these clinics is not usually considered part of secondary care, and patients referred to them are usually seen by specialist physiotherapists and GPwSIs in MSK medicine, although the composition of their staff and the services provided vary widely and might include other specialists. ${ }^{35}$ Barriers or restrictions such as criteria for referral or waiting time to these clinics are often much lower than those for direct referrals to secondary care specialities, ${ }^{35}{ }^{36}$ which might explain the high rate of such referrals. Furthermore, patients can be referred on from MSK clinics to secondary care services, such as orthopaedics or rheumatology, and this might explain the contrasting low referral rate from GPs directly to these specialities.

We examined whether GPwSI and GPs with postgraduate training in MSK/shoulder pain were more confident in making a diagnosis and whether they differed in their approach to requesting investigations or offering treatment. These GPs would be expected to have higher levels of knowledge and clinical skills in managing patients with MSK conditions. However, we found no important difference between these two groups and other GPs, neither in their confidence in making a diagnoses or the rate of investigations selected. However, with regard to treatment decisions, there were some differences between GPwSIs/ GPs who had MSK postgraduate training and the other GPs with regard to prescribing NSAIDs (less commonly) and offering corticosteroid shoulder injection (more commonly) for both patients. We had no further information about GPs' expertise nor the contents of their MSK training. However, our findings raise questions about the influence of further MSK training on GPs' clinical practice and decision making. The evidence for the influence of clinician training on clinical GP practice is inconclusive. ${ }^{37-39}$

\section{Comparison with other research}

Our findings suggest that compared with GPs in Australia, many more UK GPs selected blood tests for a patient with RCT ( $42 \%$ vs $23 \%)$ and a patient with early AdhC $(60 \%$ vs $2 \%){ }^{8}$ A larger proportion of UK GPs selected a plain radiograph of the shoulder for the AdhC patient than
Australian GPs ( $58 \%$ vs 27\%). However, USS was selected by fewer GPs in the UK, by around a third for each of our vignettes, compared with more than $80 \%$ and $90 \%$ by Australian GPs, even though there are no restrictions on requesting USS in either of these countries. With regard to treatment decisions, the only difference was that GPs in the UK were more likely than their colleagues in Australia to offer corticosteroid shoulder injection for the AdhC vignette. GPs in the UK were less likely to refer patients directly to specialists in secondary care such as orthopaedics or rheumatology than GPs in Australia, which might be explained by the high rate of referrals to MSK interface clinics in the UK. It is relevant to note that the Australian survey was conducted several years earlier, and practice and service provision might have changed since. There are no comparable figures from the UK. However, Linsell et al found in a study conducted more than 10 years earlier that $22.4 \%$ of patients with shoulder pain were referred to a specialist care within 3 years of their initial presentations.

\section{Comparing the survey two distribution methods}

Response to the postal survey was similar to previous postal surveys among GPs in the UK. ${ }^{13}{ }^{14}$ Although it yielded greater response than the online method, the postal method required more resources to administer and provided poorer quality data in relative terms, in that responses to questions were incomplete. Online survey methods have been used previously with GPs, such as invites posted on the British Medical Association or the Royal College of General Practitioners websites and direct email contact, and response rates have varied from $1.4 \%{ }^{16}$ to $40 \% .{ }^{17}$ The low response to the email method echoes similar surveys of GPs in other countries. ${ }^{404}$

\section{Strengths and limitations}

This is the first study to attempt to describe the diagnosis and management of shoulder pain patients by GPs in the UK, using a combination of general questions and questions related to clinical vignettes. Clinical vignettes have previously been shown to be a suitable method to measure aspects of clinical practice related to management decisions. ${ }^{40} 42-45$

The low response rate means that the findings are likely to be influenced by non-response bias. We could only compare responders to non-responders based on gender and location in the UK, which limits our ability to assess the likely influence of such bias. According to type of GPs responding to the survey, the $72 \%$ of responders who reported being GP principals compares with $82 \%$ of all GPs in the UK who are GP principals. ${ }^{18}$ In spite of the apparent advantages of email distribution and online data collection, the postal survey method was the more successful in terms of response. We only used direct email as the method of electronic distribution, and it is not clear whether other emerging electronic distribution methods (eg, social media) would give different response rates. 
The results might have uncovered a gap between best evidence on the diagnosis and management of shoulder pain and current GP practice. The caveat to this is that clinical vignettes are brief and can be interpreted differently by different clinicians, and the diagnosis of shoulder pain presentations is known to be challenging with symptoms and signs potentially reflecting various underlying conditions. However, the clinical vignettes used in this survey were adapted from three previous surveys in other countries. ${ }^{6-8}$ It has to be assumed that at least some of the uncertainty regarding the diagnoses among responders reflects the level of information provided in these vignettes. However, the most common cause of shoulder pain in primary care by far is rotator cuff disease followed by adhesive capsulitis. GPs, therefore, will use this pattern from their own clinical practice as they formulate their diagnostic liklihood and focus on the most common diagnoses first, but of course with an open mind to ensure not missing more serious pathologies which are rarer.

We have no direct evidence to suggest that responders to our study had access to the previous studies: no information regarding the previous studies was provided to survey participants, nor did any of the responses to our survey include any reference to these studies.

\section{Implications for future research}

The survey describes the diagnosis and treatment decisions of a large sample of GPs in the UK, for patients with shoulder pain and provides insights into the reasons for their decisions about imaging and treatment. More in-depth understanding of their clinical reasoning, perhaps using qualitative research, would particularly help better understand the rationale for their decision regarding investigations and other management decisions, as well as explore the apparent differences in responses depending on the methodology used, that is, whether the survey question was asked generally or specifically in the context of a clinical vignette.

Future research needs to investigate the impact of GPs' decisions on patient outcomes and healthcare use in longitudinal research. The data reported here could also be compared with data from general practice medical records and from studies that include patient reported data about investigations and treatments. Comparing diagnostic confidence and management decisions by GPs with other clinicians involved in managing shoulder pain, such as physiotherapists, rheumatologists and orthopaedic surgeons, would also be of interest.

\section{CONCLUSIONS}

The results of this survey among GPs in the UK using brief clinical vignettes show apparent frequent use of investigations, particularly blood tests and plain radiographs, in the management of common shoulder pain presentations. While there was a wide variation in the selected treatments, the most common were NSAIDs and physiotherapy. However, the low response means that caution needs to be exercised in generalising the findings.

Correction notice This paper has been amended since it was published Online First. Owing to a scripting error, some of the publisher names in the references were replaced with 'BMJ Publishing Group'. This only affected the full text version, not the PDF. We have since corrected these errors and the correct publishers have been inserted into the references.

Acknowledgements We would like to acknowledge all the GPs who responded to the survey. We also would like to acknowledge the contributions to the study design and delivery by the academic GPs at the Research institute: Susan Hill, Jamie Garner, Milisa Bucknall, Victoria Harper, Charlotte Purcell, Alicia Bratt and Sarah Lawton.

Contributors MA led writing the article, and DAvdW, EKA, RB, LSC, AH, ER and NEF contributed extensively to writing and finalising the manuscript.

Funding This study was funded by the Arthritis Research UK Primary Care Centre of Excellence Grant (grant number 20202). MA is funded through an NIHR Clinical Lectureship in General Practice. RB is funded by an Australian National Health and Medical Research Council (NHMRC) Senior Principal Research Fellowship. NEF, an NIHR Senior Investigator, is funded through an NIHR Research Professorship (NIHR$\mathrm{RP}-011-015)$. The views expressed are those of the authors and not necessarily those of the NHS, the NIHR or the Department of Health.

Competing interests None declared.

Ethics approval The study has been approved by the University of Keele Ethical Review Panel, Staffordshire (Reference: ERP223). Implied consent to participate in the study was obtained from all general practitioners returning a completed questionnaire. Data are maintained that they cannot be linked with identifiable participants and are anonymised for data analysis and reporting.

Provenance and peer review Not commissioned; externally peer reviewed.

Data sharing statement The authors give permission to consider data sharing of the original research article.

Open Access This is an Open Access article distributed in accordance with the Creative Commons Attribution Non Commercial (CC BY-NC 4.0) license, which permits others to distribute, remix, adapt, build upon this work non-commercially, and license their derivative works on different terms, provided the original work is properly cited and the use is non-commercial. See: http://creativecommons.org/ licenses/by-nc/4.0/

(c) Article author(s) (or their employer(s) unless otherwise stated in the text of the article) 2017. All rights reserved. No commercial use is permitted unless otherwise expressly granted.

\section{REFERENCES}

1. Urwin M, Symmons D, Allison T, et al. Estimating the burden of musculoskeletal disorders in the community: the comparative prevalence of symptoms at different anatomical sites, and the relation to social deprivation. Ann Rheum Dis 1998;57:649-55.

2. Luime JJ, Koes BW, Hendriksen IJ, et al. Prevalence and incidence of shoulder pain in the general population; a systematic review. Scand J Rheumatol 2004;33:73-81.

3. Linsell L, Dawson J, Zondervan K, et al. Prevalence and incidence of adults consulting for shoulder conditions in UK primary care; patterns of diagnosis and referral. Rheumatology 2006;45:215-21.

4. Greving K, Dorrestijn O, Winters JC, et al. Incidence, prevalence, and consultation rates of shoulder complaints in general practice. Scand $J$ Rheumatol 2012;41:150-5.

5. Jordan KP, Kadam UT, Hayward R, et al. Annual consultation prevalence of regional musculoskeletal problems in primary care: an observational study. BMC Musculoskelet Disord 2010;11:144.

6. Glazier RH, Dalby DM, Badley EM, et al. Management of common musculoskeletal problems: a survey of Ontario primary care physicians. CMAJ 1998;158:1037-40.

7. Loebenberg MI, Rosen JE, Ishak C, et al. A survey of decisionmaking processes in the treatment of common shoulder ailments among primary care physicians. Bull Hosp Jt Dis 2006;63:137-44.

8. Buchbinder R, Staples MP, Shanahan EM, et al. General practitioner management of shoulder pain in comparison with rheumatologist 
expectation of care and best evidence: an Australian national survey. PLoS One 2013;8:e61243.

9. Hegedus EJ, Cook C, Lewis J, et al. Combining orthopedic special tests to improve diagnosis of shoulder pathology. Phys Ther Sport 2015;16:87-92.

10. Lewis J. Rotator cuff related shoulder pain: assessment, management and uncertainties. Man Ther 2016;23:57-68.

11. Ottenheijm RP, Hesselmans NJ, Kemper A, et al. GPs' perspectives on the diagnostic work-up in patients with shoulder pain: a qualitative study. J Eval Clin Pract 2014;20:239-45.

12. Shah R, Edgar DF, Evans BJ. A comparison of standardised patients, record abstraction and clinical vignettes for the purpose of measuring clinical practice. Ophthalmic Physiol Opt 2010;30:209-24.

13. Cottrell E, Roddy E, Rathod T, et al. Maximising response from GPs to questionnaire surveys: do length or incentives make a difference? BMC Med Res Methodol 2015;15:3.

14. Bishop A, Foster NE, Thomas E, et al. How does the self-reported clinical management of patients with low back pain relate to the attitudes and beliefs of health care practitioners? A survey of UK general practitioners and physiotherapists. Pain 2008;135:187-95.

15. Wright KB. Researching Internet-Based populations: advantages and disadvantages of Online Survey Research, Online Questionnaire Authoring Software packages, and web survey Services. J Comput Mediat Commun 2005;10:00.

16. Smith C, Pettigrew LM, Seo HN, et al. Combining general practice with international work: online survey of experiences of UK GPs. JRSM Short Rep 2012;3:1-8.

17. National survey of GPs. 2015. The Future of General Practice 2015: British Medical Association. www.bma.org.uk/collective-voice/ committees/general-practitioners-committee/gpc-surveys/future-ofgeneral-practice. A report by ICM on behalf of the BMA.

18. The BMA, General practice in the UK. www.bma.org.uk/ pressbriefinggeneralpracticeintheuk july2014 v2.pdf

19. Binleys: NHS Directories, Mailing Lists and Public Sector Contacts. http://www.binleys.com/ (accessed 06 Jun 2017).

20. Survey Monkey company. https://www.surveymonkey.com/ (accessed 06 Jun 2017)

21. NHS in numbers. http://www.nuffieldtrust.org.uk/nhs-numbers-0.

22. Royal College of Radiologists. making the best use of clinical radiology services: referral guidelines. London: The Royal College of Radiologists, 2007. http://www.rcr.ac.uk

23. Hanchard N, Goodchild L, Thompson J, et al; Evidence-based clinical guidelines for the diagnosis, assessment and physiotherapy management of contracted (frozen) shoulder v.1.6, 'standard' physiotherapy: Endorsed by the Chartered Society of Physiotherapy, 2011. www.csp.org.uk/skipp

24. British Elbow and Shoulder Society (BESS). Pathway guidelines for sub189 acromial shoulder pain, 2013. http://.www.bess.org.uk

25. Clinical KnowledgeSummaries. http://www.cks.nhs.uk/shoulder pain

26. Diercks R, Bron C, Dorrestijn O, et al. Guideline for diagnosis and treatment of subacromial pain syndrome: a multidisciplinary review by the dutch Orthopaedic Association. Acta Orthop 2014;85:314-22.

27. Zreik NH, Malik RA, Charalambous CP. Adhesive capsulitis of the shoulder and diabetes: a meta-analysis of prevalence. Muscles Ligaments Tendons J 2016;6:26-34.
28. Hanchard NCA, Lenza M, Handoll HHG, et al. Physical tests for shoulder impingements and local lesions of Bursa, tendon or labrum that may accompany impingement (Review). The Cochrane Library $2013 \mathrm{http}: / /$ www.thecochranelibrary.com

29. Cullen DM, Breidahl WH, Janes GC. Diagnostic accuracy of shoulder ultrasound performed by a single operator. Australas Radiol 2007;51:226-9.

30. Judge A, Murphy RJ, Maxwell R, et al. Temporal trends and geographical variation in the use of subacromial decompression and rotator cuff repair of the shoulder in England. Bone Joint $J$ 2014;96B:70-4.

31. Buchbinder R, Green S, Youd JM, et al. Oral steroids for adhesive capsulitis. Cochrane Database Syst Rev 2006:CD006189.

32. Buchbinder R, Green S, Youd JM, et al. Arthrographic distension for adhesive capsulitis (frozen shoulder). Cochrane Database Syst Rev 2008:CD007005.

33. Page MJ, Green S, McBain B, et al. Manual therapy and exercise for rotator cuff disease. Cochrane Database of Systematic Reviews 2016. CD012224

34. Page MJ, Green S, Kramer S, et al. Manual therapy and exercise for adhesive capsulitis (frozen shoulder). Cochrane Database of Systematic Reviews 2014. CD011275.

35. Roberts C, Dolman EA, Adebajo AO, et al. A national qualitative survey of community-based musculoskeletal services in the UK. Rheumatology 2003;42:1074-8.

36. Roddy E, Zwierska I, Jordan KP, et al. Musculoskeletal clinical assessment and treatment services at the primary-secondary care interface: an observational study. Br J Gen Pract 2013;63:141-8.

37. Watson J, Helliwell P, Morton V, et al. Shoulder acute pain in primary healthcare: is retraining effective for GP principals? SAPPHIRE-a randomized controlled trial. Rheumatology 2008;47:1795-802.

38. Petrella RJ, Davis P. Improving management of musculoskeletal disorders in primary care: the Joint Adventures Program. Clin Rheumatol 2007;26:1061-6.

39. Tu K, Davis D. Can we alter physician behavior by educational methods? lessons learned from studies of the management and follow-up of hypertension. J Contin Educ Health Prof 2002;22:11-22.

40. Braithwaite D, Emery J, De Lusignan S, et al. Using the internet to conduct surveys of health professionals: a valid alternative? Fam Pract 2003;20:545-51.

41. Crouch S, Robinson P, Pitts M. A comparison of general practitioner response rates to electronic and postal surveys in the setting of the National STI Prevention Program. Aust NZ J Public Health 2011;35:187-9.

42. Luck J, Peabody JW, Dresselhaus TR, et al. How well does chart abstraction measure quality? A prospective comparison of standardized patients with the medical record. Am J Med 2000;108:642-9.

43. Peabody JW, Luck J, Glassman P, et al. Comparison of vignettes, standardized patients, and chart abstraction: a prospective validation study of 3 methods for measuring quality. JAMA 2000;283:1715-22.

44. Veloski J, Tai S, Evans AS, et al. Clinical vignette-based surveys: a tool for assessing physician practice variation. Am J Med Qual 2005;20:151-7.

45. Shah R, Edgar D, Evans BJ. Measuring clinical practice. Ophthalmic Physiol Opt 2007;27:113-25. 